\title{
Sistemas de producción en áreas con cangahua habilitada en la Sierra Norte de Ecuador
}

\section{Production systems in areas with reclaimed cangahua soils in the Northern Highlands of Ecuador}

\author{
Jaime Hidrobo ${ }^{1}$, Mathilde da Costa ${ }^{2}$, Christian Prat ${ }^{1,3,4}$, Germán Trujillo ${ }^{l}$, Julio Moreno ${ }^{4}$, Carlos Alberto Ortega \\ 'Universidad Central del Ecuador. Facultad de Ciencias Agrícolas. Jerónimo Leiton y Av. La Gasca s/n. Ciudadela Universitaria. 170521 Quito, \\ Ecuador \\ ${ }^{2}$ Ecole Supérieure d'Agro-Développement International (ISTOM), Cergy, Francia \\ ${ }^{3}$ Programa "Prometeo"- SENESCYT \\ ${ }^{4}$ Instituto Espacial Ecuatoriano
}

\begin{abstract}
Resumen
La presente investigación tiene como objetivo caracterizar las experiencias agroecológicas de los campesinos que poseen lotes con cangahuas recuperadas, situados en una pequeña región del callejón interandino ecuatoriano, específicamente del cantón Cayambe. Las cangahuas son tobas volcánicas estériles que cubren amplias áreas de este sector y su rehabilitación y manejo presentan varios desafíos ambientales, sociales y económicos que frenan considerablemente el desarrollo agrícola de esta zona. La habilitación de la cangahua abre la posibilidad de dinamizar la deprimida agricultura característica de las zonas donde ha aflorado este sustrato. El estudio de la gestión de las parcelas recuperadas revela una homogeneidad global entre los sistemas de producción. Las diferencias observables pueden ser explicadas por el poco apoyo financiero y técnico, y por la limitación de acceso al agua. La conversión de las cangahuas a tierras agrícolas y sobre ellas la implementación de parcelas con manejo agroecológico podría responder a las expectativas de los campesinos y hacer frente a los problemas ambientales.
\end{abstract}

Palabras clave: cangahuas, suelo, erosión, sistemas agrícolas, agroecología, habilitación agrícola.

\begin{abstract}
This investigation was conducted to characterize the experiences in agro ecological production used by farmers on reclaimed cangahua soils in a small inter-Andean region from the Cayambe Township, in the Ecuadorian sierra. The cangahuas are sterile volcanic tuffs which cover huge surfaces of this area, and its reclamation and management pose many environmental, social and economic issues which hinder considerably the agricultural development in this area. Nonetheless, cangahua rehabilitation offers the possibility of revitalizing the depressed agriculture of this zone. The evaluation of the management practices of the recovered plots highlights the existence of an overall homogeneity in the production systems. The observed differences can be explained by the poor financial and technical support, and by limited access to water resources. Cangahua conversion into agricultural land and the implementation of fields with agro ecological management could meet the farmer's needs and address environmental issues.
\end{abstract}

Keywords: cangahuas, soil, erosion, farming systems, agroecology, reclaimation. 


\section{Introducción}

Las cangahuas son rocas conformadas por materiales piroclásticos que normalmente fueron cubiertas por cenizas volcánicas recientes sobre las cuales se desarrolló un suelo profundo y fértil. En Ecuador, las cangahuas están presentes en las estribaciones de los Andes de la Sierra Norte en un área aproximada de 270000 ha, estimándose que las cangahuas han aflorado en 83000 ha después de que el suelo superficial se ha erosionado. Las cangahuas expuestas son duras y tienen muy bajo contenido de nitrógeno $(\mathrm{N})$ y materia orgánica (MO), razón por la cual no se pueden utilizar para la producción agrícola (Zebrowski et al., 1997). Sin embargo, después de aflojarlas por medio de trabajo manual o con maquinaria pesada, algunos campesinos lograr habilitar la cangahua y cultivarla con el uso limitado de insumos (Zebrowsky y Azucena, 1996).

Actualmente, el Gobierno Nacional, a través del Ministerio de Agricultura Ganadería y Pesca (MAGAP), entrega maquinaria pesada para recuperación de cangahuas a prefecturas y municipios del país mediante el Programa Nacional de Habilitación Agrícola de Cangahuas. Sin embargo, el trabajo de habilitación se conduce sin poner en práctica las mejores estrategias técnicas, agronómicas y sociales requeridas para la recuperación exitosa del material endurecido. Estos procesos son costosos tanto para el país así como para los mismos campesinos.

Si bien es cierto que se han realizado muchos estudios en el ámbito de rehabilitación de cangahuas en las décadas de los años 80 y 90 (de Noni, Viennot, Asseline, \& Trujillo, 2001; Zebrowski, 1997), es necesario actualizarlos y complementarlos ya que muchos aspectos relacionados con este tema no fueron tratados durante esos años. Además, se carece actualmente de información sobre la descripción y evaluación de los sistemas de producción agrícola que ubicados en las áreas degradas por erosión en la Sierra de Ecuador, y menos aún en el caso áreas donde han aflorado las cangahuas.

Teniendo en cuenta las condiciones anteriormente descritas, la Universidad Central del Ecuador propuso, dentro de sus líneas de investigación, un proyecto de caracterización de las cangahuas y de los métodos para su rehabilitación en el contexto del Cambio de Matriz Productiva y de la Seguridad Alimentaria del país, bajo un enfoque de producción agroecológica. El presente trabajo se desarrolló como parte de este proyecto y tiene como objetivo caracterizar las experiencias agrícolas de campesinos que tienen parcelas que poseen cangahuas habilitadas en comunidades del cantón $\mathrm{Ca}$ yambe, provincia de Pichincha.

El estudio se condujo en la parroquia Cangahua, ubicada a $13 \mathrm{~km}$ al sur de la ciudad de Cayambe, capital del cantón Cayambe. Esta parroquia con sus $329 \mathrm{~km}^{2}$, representa el $30 \%$ de la superficie total del cantón y de sus 16200 habitantes, el 63\% se dedica a la producción agrícola (INEC, 2010).

La zona de estudio está ubicada en la cuenca del río Pisque. Los procesos geológicos y tectónicos son responsables de la presencia de una topografía irregular, existen extensos valles, altas terrazas, lenguas, domos y picos, en muchos casos con pronunciadas pendientes $(>50 \%)$.

La temperatura promedio anual del cantón, registrada en la Estación Meteorológica de Cayambe ubicada a 2,840 m.s.n.m, es de $14.1^{\circ} \mathrm{C}$, pero existe un amplio rango entre temperaturas diurnas y nocturnas que pueden variar de $20{ }^{\circ} \mathrm{C}$ durante el día a $6{ }^{\circ} \mathrm{C}$ durante la noche Sin embargo, las diferencias topográficas de la zona generan contrastes climatológicos muy marcados y globalmente se pueden distinguir 4 niveles altitudinales que caracterizan la temperatura:

- $\quad$ Piso de 2,600 a 2,800 m.s.n.m., temperatura promedio anual entre 13 y $22{ }^{\circ} \mathrm{C}$

- $\quad$ Piso de 2,800 a 3,600 m.s.n.m., temperatura promedio anual entre 10 y $13{ }^{\circ} \mathrm{C}$

- $\quad$ Piso de 3,600 a 4,200 m.s.n.m., temperatura promedio anual entre 0 y $10{ }^{\circ} \mathrm{C}$

- $\quad$ Piso $>4,200$ m.s.n.m., temperatura promedio anual alrededor de $0{ }^{\circ} \mathrm{C}$

La lluvia se distribuye a lo largo del año en un patrón sencillo: una temporada seca (junio a agosto) y una temporada de lluvias (septiembre a mayo). Octubre y abril corresponden a los meses más húmedos del año (Figura 1). Las lluvias, aunque siguen un patrón parecido al de la temperatura ( $>$ altitud $>$ precipitaciones), presentan fuertes disparidades locales relacionadas a los relieves muy irregulares que generan muchos microclimas como se observa en los registros de las estaciones 
meteorológicas de cantón Cayambe que se presentan en la Tabla 1 (MIDENA, SENPLADES, IEE, \& Gobierno Autónomo Decentralizado de Pichincha, 2013).

La geología, clima, relieve y el uso antrópico del suelo ha promovido la formación de distintos tipos de suelos que se describen brevemente a continuación (IUSS Working Group WRB, 2014; PRONAREG-ORSTOM, 1982):

- $\quad$ A altitudes inferiores a los 2,500 m.s.n.m., en las riberas de los ríos Pisque y Guachalá, se encuentran suelos arenosos clasificados como Fluvisoles.

- $\quad$ Entre 2,500 y 3,600 m.s.n.m. se encuentran suelos clasificados como Molisoles, de alto contenido de $\mathrm{MO}$ y que a menudo presentan un horizonte de acumulación de arcilla sumamente compacta que genera problemas de circula- ción de agua en el perfil. Estos suelos de espesor variable son los que recubren las cangahuas.

- En las áreas erosionadas a nivel de los piedemontes, también ubicadas entre 2,500 y 3,600 m.s.n.m., aparecen las cangahuas, particularmente en las rupturas de las pendientes. Este es el caso de la parroquia Cangahua, cantón Cayambe.

- $\quad$ Entre los 3,600 hasta los 4500 m.s.n.m. se encuentran los páramos donde predominan Andosoles desarrollados en un régimen per-húmedo de humedad del suelo, generalmente muy profundos y con capacidad de retener enormes cantidades de agua ( 3 a 4 veces su peso seco).

- $\quad$ Por arriba de los 4,500 m.s.n.m. se encuentran únicamente rocas poco evolucionadas (Regosoles) y luego el dominio del glaciar (Cryosoles).

Tabla 1. Ubicación y pluviometría anual (series climáticas 1985-2009) de cinco estaciones meteorológicas del cantón Cayambe (MIDENA et al., 2013).

\begin{tabular}{llllll}
\hline Código & Nombre estación & \multicolumn{2}{c}{ Coordenadas } & Altitud (m.s.n.m). & Pluviometría anual (mm) \\
& & Este & Norte & \\
\hline \multirow{2}{*}{ M023 } & Olmedo-Pichincha & 825692 & 1001569 & 3,120 & 820 \\
M344 & Cangahua & 815332 & 9993576 & 3,140 & 641 \\
M359 & Cayambe & 818117 & 1000560 & 2,840 & 941 \\
M566 & Ascázubi INAMHI & 801828 & 9991375 & 2,580 & 796 \\
M628 & Hda. Pesillo & 826871 & 1001756 & 3,160 & 734 \\
\hline
\end{tabular}

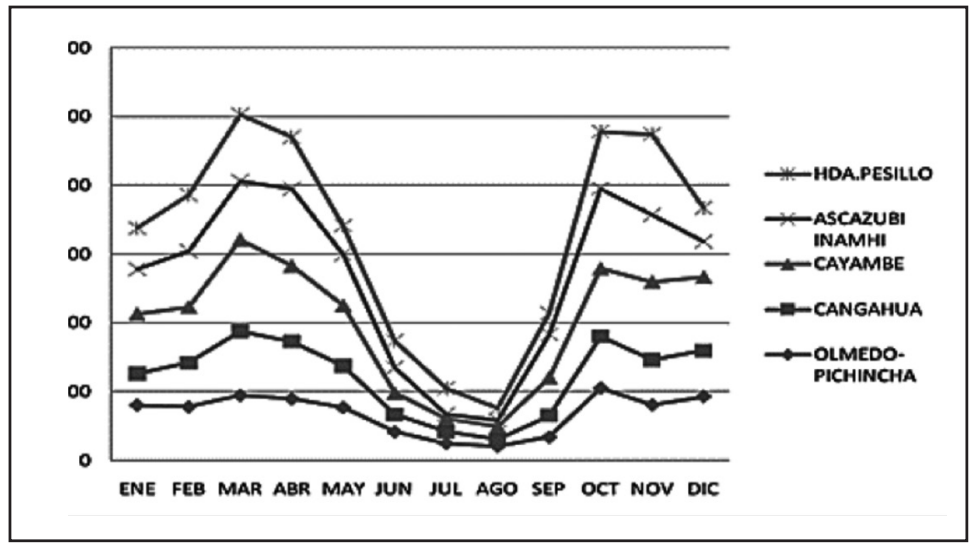

Figura 1. Precipitaciones medias mensuales de 5 estaciones Meteorológicas (Series climáticas 1985-2009) del cantón Cayambe (MIDENA et al., 2013). 
De igual forma, la vegetación se distribuye de acuerdo al relieve, tipo de suelo y clima. De acuerdo a la clasificación de las zonas de vida de Holdridge, la zona donde aparecen las cangahuas corresponde al "bosque seco de sierra baja" (Holdridge, 1969). La vegetación es una mezcla de vegetación arbustiva (espontánea y cultivada) y de cultivos. Existen bosques de eucaliptos y a veces de pinos. Los árboles nativos se encuentran en las quebradas y alrededor de los lotes agrícolas. En las áreas más degradadas es común encontrar el penco negro (Agave americana L.).

\section{Materiales y métodos}

Los objetivos del estudio fueron: 1) Determinar cuáles son los sistemas de producción establecidos en la zona de influencia del proyecto y 2) Evaluar las diferentes maneras de habilitación de lotes con cangahua utilizadas en la zona y su manejo agronómico. Este trabajo comenzó con una extensa búsqueda bibliográfica de la información local e internacional sobre cangahuas y con la organización de encuentros con las autoridades cantonales y parroquiales, con la ONG Agrónomos y Veterinarios Sin Fronteras (AVSF) que trabaja en esta área desde el año 2013 y con líderes de la comunidad. Se organizaron visitas a lotes de campesinos y productores con representantes de estas organizaciones. Además, con el apoyo del Instituto Espacial Ecuatoriano (IEE) se obtuvieron los mapas detallados de la topografía de la zona, así como los mapas generales de suelos y los de afloramientos de cangahua.

Después de haber obtenido la información básica sobre la zona de intervención se procedió a elaborar encuestas con un cuestionario diseñado para obtener la información requerida por los objetivos del estudio. Las encuestas fueron respondidas por las autoridades locales, administradores zonales, personal de las ONG y naturalmente los pequeños productores y campesinos de la zona. Las encuestas de campo se desarrollaron en 6 de las 8 comunidades de la parroquia Cangahua (Buena Esperanza, Cuniburo, Pitaná Alto, Pitaná Bajo, Santa Marianita de Pingulmi y Santa Rosa de Pingulmi). Las encuestas se realizaron directamente en las parcelas con los dueños o usuarios. La entrevista, de tipo semi directivo, tuvo una duración de media hora. La localización de cada una de las parcelas evaluadas se referenció con GPS. Los criterios utilizados en las encuestas fueron los siguientes:
- Sistemas de producción: presencia o ausencia de sistemas de riego, tipo de tratamiento fitosanitario (biológico, mezclado, químico o ninguno), tipo de fertilizante (orgánico, mixto, química o ninguno), rotación de cultivos y cultivos intercalados.

- Habilitación agrícola de cangahuas y su protección: tenencia de la tierra, edad de habilitación de las cangahuas, forma de rehabilitación y colaboradores (instituciones públicas, organizaciones no gubernamentales), métodos de conservación y manejo del suelo.

Los datos recolectados en la matriz de la encuesta se analizaron de acuerdo a la metodología de cuadros dinámicos y de análisis de componente múltiple. En el primer caso se creó una función de concatenación entre 5 criterios escogidos para definir los sistemas de producción y el segundo se utilizó programa STATA 10 para analizar los procesos de habilitación de cangahuas y los métodos de conservación.

\section{Resultados}

\subsection{Localización de las cangahuas}

Sobre el mapa de suelos elaborado por PRONAREG-ORSTOM (1982) y digitalizado por el Instituto Espacial Ecuatoriano (IEE) se seleccionaron las zonas donde las cangahuas han aflorado completamente o en forma parcial para definir las zonas de intervención del proyecto en la parroquia Cangahua. Basándose en esta información se decidió elegir las áreas con afloramiento de cangahua ubicadas entre 2,600 y 3,200 m.s.n.m. y con pendientes menores a $40 \%$ como zonas susceptibles a ser habilitada para agricultura. La superficie que cumplió con estos criterios cubre un área de 1,623 ha, de las cuales, 351 ha (21\%) son lotes abandonados (Figura 2) que, en general, son parcelas aisladas que se encuentran lejos de las viviendas, de difícil acceso, sin riego y sumamente erosionadas. Cuando logra crecer vegetación, ésta está compuesta principalmente por pencos y en algunos sitios se encuentran bosques de eucalipto. Estas zonas fueron excluidas del posible proceso de rehabilitación. Las 1,272 ha restantes corresponden a zonas con cangahuas que potencialmente pueden ser habilitadas para agricultura. 


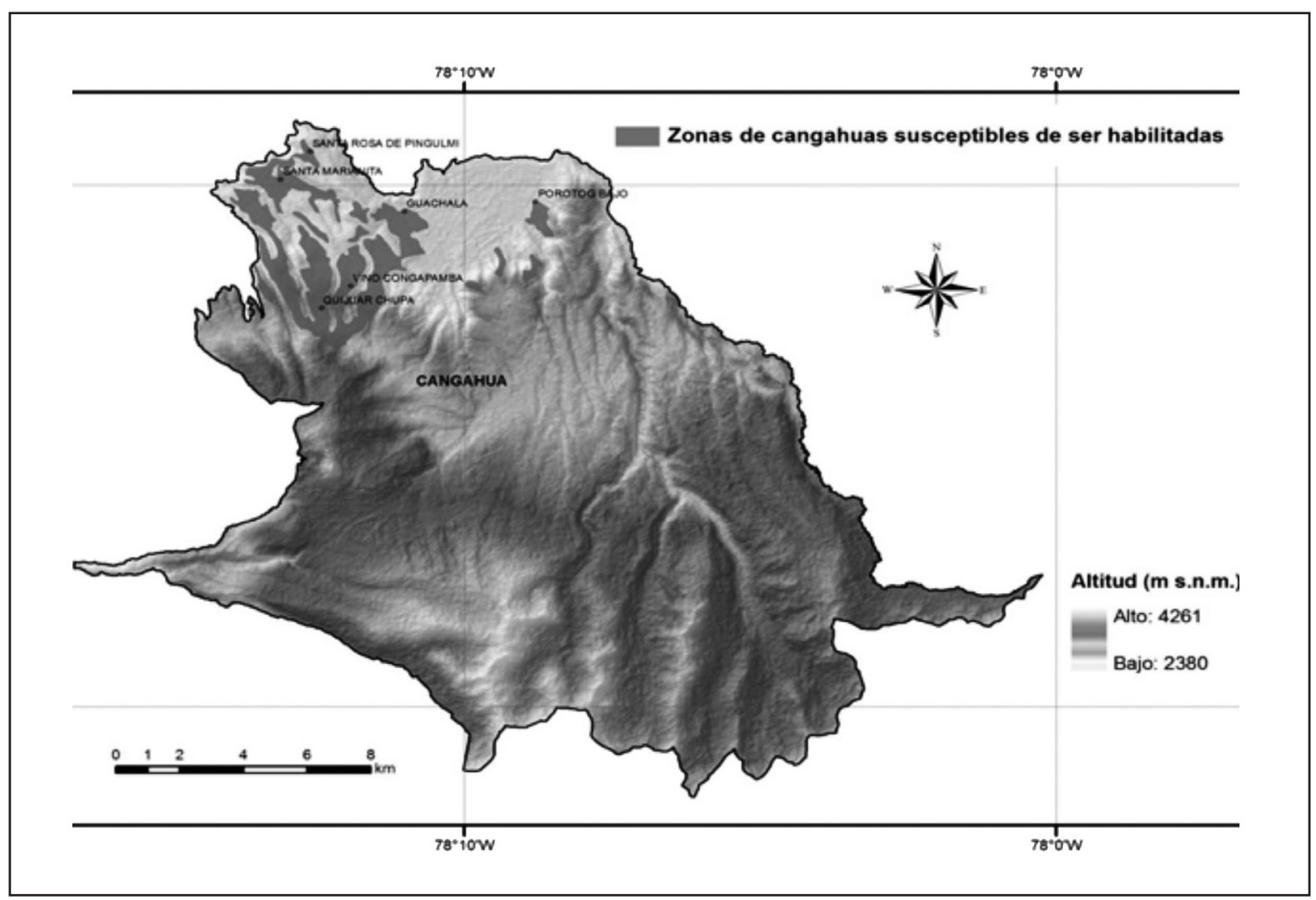

Figura 2. Ubicación geográfica de las zonas con cangahuas susceptibles de ser habilitadas.

Fuente: MAGAP, 2012 \& MAGAP-GEOPORTAL, 2013)

\subsection{Tipología de los Productores}

Inicialmente se entrevistaron 75 familias pertenecientes a 6 de las 8 comunidades de la parroquia de Cangahua, pero se conservaron finalmente 65 familias para procesar los resultados. Cabe destacar que $95 \%$ de las personas encuestadas fueron mujeres debido a que la mayoría de los hombres trabaja fuera de la comunidad, en particular en empresas floriculturas del cantón. Los hombres apoyan al trabajo de campo los fines de semana y durante periodos de mayor actividad (siembra y cosecha), mientras que las mujeres se dedican al campo todos los días del año al mismo tiempo que atienden las tareas domésticas.

La estructura de los hogares entrevistados corresponde a familias de 3 a 5 hijos, a menudo con una diferencia significativa de edad entre los ellos. En general, las mujeres tienen un nivel de educación primaria, a diferencia de sus esposos quienes tienen educación secundaria. Por otra parte, los hijos mayores continúan con educación superior y por esta razón tuvieron que dejar el campo y migrar a las ciudades (incluyendo Quito). El aumento del nivel de educación se traduce en una mejor situación económica de los habitantes de la zona. Sin embargo, esta condición también está asociada con el abandono de la tierra cultivada ancestralmente por sus padres. Las nuevas generaciones que salieron a estudiar en las ciudades pierden interés en el trabajo del campo y prefieren quedarse en las zonas urbanas para continuar con su vida.

Los datos demostraron que los productores de la zona donde aflora la cangahua se concentran en dos grandes grupos basándose en la disponibilidad de riego. En efecto, la presencia o no del agua se traduce por un modo de gestión de la tierra completamente distinto. En los lotes sin riego, los cultivos son temporales, principalmente monocultivos, mientras que en los lotes con riego existen cultivos asociados (Tabla 2). Todos los productores tienen animales, una vaca y/o caballo, con o sin ovejas y/o un chancho, pero casi todos tienen cuyes y gallinas. Por esta razón se determinó que éste no es un elemento discriminatorio. 
Tabla 2. Tipología de productores en zonas con afloramiento de cangahuas.

\begin{tabular}{|c|c|c|c|c|c|c|}
\hline & $\begin{array}{l}\text { Categorías } \\
\text { de personas }\end{array}$ & $\begin{array}{l}\text { Actividad } \\
\text { agrícola principal }\end{array}$ & $\begin{array}{l}\text { Superficie } \\
\text { cultivada }\end{array}$ & $\begin{array}{c}\text { Destino } \\
\text { del cultivo }\end{array}$ & $\begin{array}{c}\text { Con tierras } \\
\text { degradadas } \\
\text { o habilitadas } \\
\end{array}$ & $\begin{array}{c}\text { Proporción } \\
\%\end{array}$ \\
\hline \multirow[t]{4}{*}{ SIN RIEGO } & $\begin{array}{l}\text { Pequeños agricul- } \\
\text { tores con animales }\end{array}$ & $\begin{array}{l}\text { Monocultivos } \\
\text { (cereales-forrajes) }\end{array}$ & $<1$ ha & Venta & $\mathrm{Si}$ & 93 \\
\hline & $\begin{array}{l}\text { Pequeños agricul- } \\
\text { tores con animales }\end{array}$ & $\begin{array}{l}\text { Asociación } \\
\text { (maíz/fréjol/legumi- } \\
\text { nosa) }\end{array}$ & $<1$ ha & $\begin{array}{l}\text { Venta y auto- } \\
\text { consumo }\end{array}$ & $\mathrm{Si}$ & 5,5 \\
\hline & $\begin{array}{l}\text { Medianos agricul- } \\
\text { tores con animales }\end{array}$ & $\begin{array}{l}\text { Monocultivos } \\
\text { (maíz-forrajes-legu- } \\
\text { minosas) }\end{array}$ & 1 a 4 ha & $\begin{array}{l}\text { Venta y auto- } \\
\text { consumo }\end{array}$ & $\mathrm{Si}$ & 1,5 \\
\hline & & & & & & 100 \\
\hline \multirow[t]{4}{*}{ CON RIEGO } & $\begin{array}{l}\text { Pequeños agricul- } \\
\text { tores con animales }\end{array}$ & $\begin{array}{l}\text { Agricultura Diversi- } \\
\text { ficada (cereales-le- } \\
\text { guminosas-frutas-le- } \\
\text { gumbres-forrajes) }\end{array}$ & 0.01 a 0.3 ha & $\begin{array}{l}\text { Autoconsumo } \\
\text { y a veces venta }\end{array}$ & $\mathrm{Si}$ & 15 \\
\hline & $\begin{array}{l}\text { Pequeños agricul- } \\
\text { tores con animales }\end{array}$ & $\begin{array}{l}\text { Asociación (maíz/ } \\
\text { fréjol - legumino- } \\
\text { sas-quinua) }\end{array}$ & $<1$ ha & $\begin{array}{l}\text { Autoconsumo } \\
\text { y a veces venta }\end{array}$ & $\mathrm{Si}$ & 67 \\
\hline & $\begin{array}{l}\text { Pequeños agricul- } \\
\text { tores con animales }\end{array}$ & $\begin{array}{l}\text { Monocultivos } \\
\text { (cereales-forraje) }\end{array}$ & $<1$ ha & $\begin{array}{l}\text { Autoconsumo } \\
\text { y a veces venta }\end{array}$ & Propia & 18 \\
\hline & & & & & & 100 \\
\hline
\end{tabular}

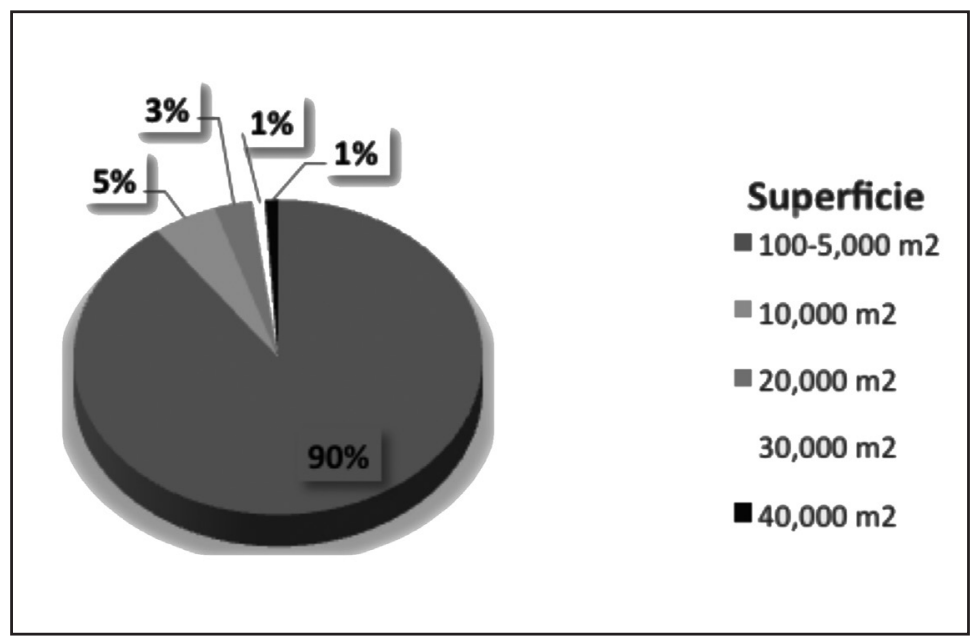

Figura 3. Superficie de lotes con cangahuas degradadas o habilitadas.

Una característica determinante de las condiciones de estas familias es que las parcelas son muy pequeñas. El 89\% la superficie de los lotes degradados o habilitados no supera el área de una hectárea, el lote más grande fue de 4 ha y la media general del tamaño de los lotes de 0.6 ha (Figura $3)$. Aun si se añaden las tierras que no están erosionadas ( $\sin$ presencia de cangahua), la superficie de los lotes sigue siendo pequeña, por esta razón los campesinos no pueden sobrevivir solamente de la producción agrícola. El ingreso económico externo conseguido con el trabajo del hombre permite que la familia pueda satisfacer las necesidades básicas. La producción agrícola de los lotes sirve primero para auto-consumo y el sobrante se comercializa. Sin embargo, el principal destino de los cereales es la venta en los mercados locales o entre vecinos (Figura 4). 


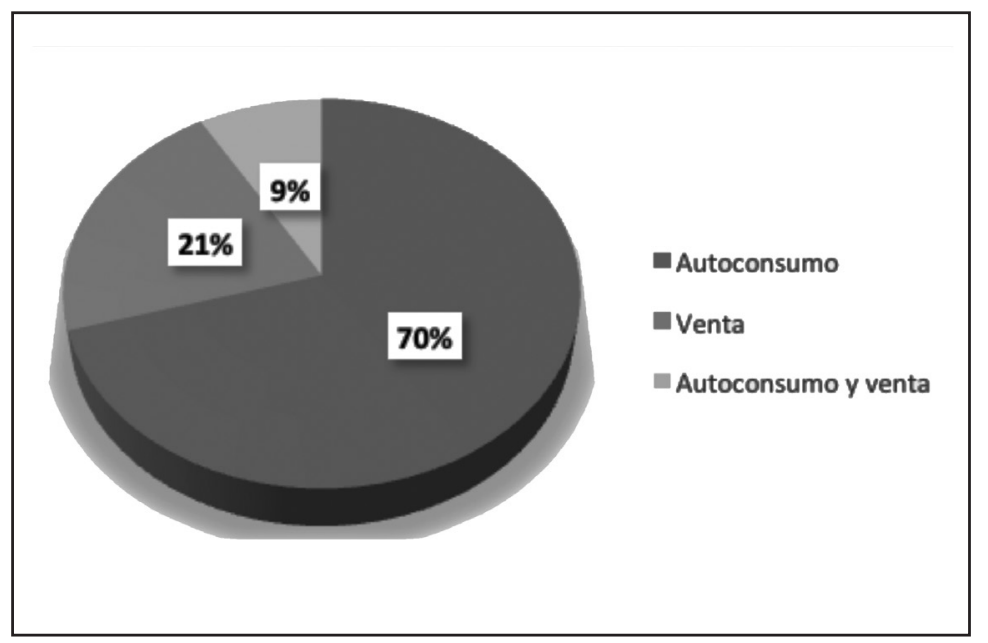

Figura 4. Destino de la producción de la zona en estudio.

\subsection{Tipos de cultivos}

Los principales cultivos de los lotes incluidos en el estudio son cereales (trigo, maíz, cebada y avena), hortalizas (col, coliflor, cebolla, brócoli, zanahoria, alverja, haba y fréjol), cultivos andinos (chocho y quinua) y frutales (aguacate, tomate de árbol y chirimoya). Algunas personas han invertido en invernaderos para cultivos comerciales como tomate y flores. Pocos campesinos cultivan papas debido a que la tierra erosionada tiene baja fertilidad y los rendimientos son precarios. Los agricultores prefieren cultivar papas en lotes ubicados sobre los 3,600 m.s.n.m., sitios donde la tierra es fértil, pero estas zonas no deberían utilizarse para cultivos por ser tierras de páramo que deben ser utilizadas exclusivamente para conservación.

Como se indicó, todos los productores tienen cría de animales, por lo tanto, la presencia de plantas forrajeras, cultivadas o no, es común en la zona. Las principales especies son raygrass, alfalfa, holco, llantén, kikuyo, vicia y avena. Generalmente, los productores tienen pocos animales: vacas lecheras, cuyes, cerdos, gallinas y en ocasiones cabras u ovejas. La cría de animales es fuente de proteínas y de ingresos complementarios, así también se constituye en una fuente importante de nutrientes para mantener la fertilidad del suelo.

\subsection{Sistemas de cultivos}

Los lotes sin riego se utilizan principalmente con monocultivos o son terrenos abandonados que man- tienen una vegetación raquítica llamada localmente "chaparro". Los monocultivos son de cebada, avena, maíz o trigo. La única asociación en estos lotes es de maíz-frejol. Estos monocultivos reciben ocasionalmente fertilización mineral, pero cuando no se usan fertilizantes se utilizan los residuos de corral dejados por la crianza de animales, particularmente de bovinos y cuyes, como fuente de nutrientes. Por otro lado, el $69 \%$ de las parcelas con riego tienen asociaciones diversas de cultivos. La mayoría de los agricultores utilizan pocos pesticidas porque los cultivos no generan suficientes recursos para destinarlos a la compra de agroquímicos, pero también se debe mencionar que en la zona se produce poca papa que es uno de los cultivos más afectados por plagas y enfermedades, lo que reduce drásticamente el uso de biocidas. Se usa curacrón para luchar contra la larva de la polilla americana del maíz (Spodoptera frugiperda) y cuanto se cultiva papa se utiliza mancozeb, curacron, furadan, sursate o ridomil para controlar problemas ocasionados por insectos como las polillas (Phtorimaea operculella, Symmestrichema tangolias, Tecia solanivora), la pulguillas (Epitrix spp.), el gusano blanco (Premnotrypes spp.) y el hongo llamado comunmente lancha (Phytophthora infestans). Los agricultores no han recibido capacitación para el uso de estos productos, lo que puede provocar problemas de salud, así como contaminación ambiental. Algunos campesinos utilizan productos orgánicos como bioles y extractos de ajo, pimiento u ortiga para controlar los problemas sanitarios de los cultivos, pero la falta de capacitación en técnicas de control biológico dificultan el uso y la producción de estos materiales. Los sistemas de 
cultivo encontrados en la zona donde se condujo el estudio se presentan en la Tabla 3.

Se detectó que en las parcelas sin riego existen dos tipos de sistemas de producción agroecológicos $\left(\mathrm{N}^{\circ} 1\right.$ y 5 en la parte superior de la Tabla 3 ), los cuales se basan en el uso de productos orgánicos y en asociación de cultivos. Sin embargo, la ausencia de riego limita en gran medida las posibilidades de escoger entre muchos cultivos para mantener las asociaciones. Estos dos sistemas representan solamente $15 \%$ de las parcelas observadas, las demás son monocultivos. En las parcelas con riego se encontraron dos tipos de sistemas de cultivo agroecológico $\left(\mathrm{N}^{\circ} 1\right.$ y 5 en la parte inferior de la Tabla 3), pero en este caso representan el $74 \%$ de los lotes con uso agrícola. Además, son sistemas mucho más complejos por los tipos de asociaciones existentes. Este tipo de sistema de cultivo se describe en la Tabla 4.

Tabla 3. Sistemas agrícolas identificados en los lotes sin riego y con riego en la zona de estudio.

\begin{tabular}{|c|c|c|c|c|c|c|}
\hline \multicolumn{7}{|c|}{ CRITERIOS DE REFERENCIAS UTILIZADOS } \\
\hline & Ref. & $\begin{array}{c}\text { Producto } \\
\text { Fitosanitario }\end{array}$ & Fertilización & $\begin{array}{c}\text { Manejo } \\
\text { de Cultivos }\end{array}$ & Rotación & $\begin{array}{c}\text { Parcelas } \\
\text { Identificadas } \\
(\%)\end{array}$ \\
\hline & 1 & Orgánico & Orgánico & Asociación & $\mathrm{Si}$ & 4 \\
\hline & 2 & Mixto & Orgánico & Asociación & $\mathrm{Si}$ & 5 \\
\hline & 3 & Químico & Mixto & Monocultivo & $\mathrm{Si}$ & 5 \\
\hline S & 4 & Sin & Mixto & Monocultivo & $\mathrm{Si}$ & 5 \\
\hline $\mathbf{N}$ & 5 & $\operatorname{Sin}$ & Orgánico & Asociación & $\mathrm{Si}$ & 11 \\
\hline & 6 & $\operatorname{Sin}$ & Orgánico & Monocultivo & $\mathrm{Si}$ & 27 \\
\hline $\mathbf{R}$ & 7 & Sin & Químico & Monocultivo & No & 5 \\
\hline $\begin{array}{l}1 \\
\mathbf{E}\end{array}$ & 8 & $\operatorname{Sin}$ & Sin & Monocultivo & $\mathrm{Si}$ & 11 \\
\hline G & 9 & $\operatorname{Sin}$ & $\operatorname{Sin}$ & "Chaparro" & No & 27 \\
\hline $\mathbf{O}$ & Total & & & & & 100 \\
\hline & 1 & Orgánico & Orgánico & Asociación & $\mathrm{Si}$ & 15 \\
\hline C & 2 & Químico & Mixto & Monocultivo & No & 2 \\
\hline $\begin{array}{l}\mathbf{O} \\
\mathbf{N}\end{array}$ & 3 & Químico & Orgánico & Asociación & $\mathrm{Si}$ & 7 \\
\hline & 4 & Sin & Mixto & Asociación & $\mathrm{Si}$ & 3 \\
\hline $\mathbf{R}$ & 5 & $\operatorname{Sin}$ & Orgánico & Asociación & $\mathrm{Si}$ & 59 \\
\hline $\begin{array}{l}\text { I } \\
\mathbf{F}\end{array}$ & 6 & $\operatorname{Sin}$ & Orgánico & Monocultivo & No & 8 \\
\hline $\begin{array}{l}\text { E } \\
\text { G }\end{array}$ & 7 & Sin & Orgánico & Monocultivo & $\mathrm{Si}$ & 6 \\
\hline $\mathbf{O}$ & Total & & & & & 100 \\
\hline
\end{tabular}

Tabla 4. Sistemas agroecológicos existentes en las áreas con riego en la zona de intervención.

\begin{tabular}{|c|c|c|c|}
\hline & Características del sistema & Superficies & $\begin{array}{c}\text { Parcelas } \\
\text { Identificadas }(\%)\end{array}$ \\
\hline Sistema 1 & $\begin{array}{l}\text { - Fertilización orgánica } \\
\text { - Uso de productos fitosanitarios orgánicos } \\
\text { - Asociaciones de cultivos muy complejas con arboles } \\
\text { - Rotaciones }\end{array}$ & $\begin{array}{c}100 \\
\mathrm{a} \\
5,000 \mathrm{~m}^{2}\end{array}$ & 15 \\
\hline Sistema 2 & $\begin{array}{l}\text { - Fertilización orgánica } \\
\text { - Ausencia de productos fitosanitarios orgánicos } \\
\text { - Asociaciones de cultivos complejas } \\
\text { - Rotaciones }\end{array}$ & $\begin{array}{c}100 \\
\mathrm{a} \\
10,000 \mathrm{~m}^{2}\end{array}$ & 59 \\
\hline
\end{tabular}


El sistema 1, que es en realidad un sistema de agroforestería, fue desarrollado hace 10 años por la Fundación francesa SEDAL (Servicios para el Desarrollo Alternativo) que implementó un proyecto de agroecología a través de la organización de mujeres BIOVIDA. Aunque han salido algunos de sus miembros, esta agrupación de mujeres continua su trabajo en varias actividades comunitarias incluyendo las prácticas agroecológicas.

Además de la variedad de los cultivos y de la siembra en alta densidad para reducir la presencia de malezas y proteger el suelo de la erosión, la producción de cultivos se complementa con la ganadería. Los animales consumen el forraje cultivado en las parcelas y sus deyecciones se incorporan al suelo, junto con gallinaza comprada a productores avícolas. Los residuos de cultivos también se incorporan al suelo. Si bien es cierto que este sistema requiere mayor cuidado y mejor mantenimiento, la productividad, la variedad y la calidad de los productos cosechados hacen que sus dueños obtengan un ingreso mayor que los otros sistemas de cultivo prevalentes en la zona y, además, no tienen efectos nocivos para la salud y son amigables con el ambiente.

\subsection{Dinámica de los procesos de habilitación agrícola de las cangahuas}

Muchas parcelas sin riego fueron habilitadas para la agricultura hace varios años, pero dejaron de ser cultivadas. El mal manejo de estas parcelas, particularmente la falta de un sistema de control de erosión luego de la habilitación de las cangahuas o la falta de mantenimiento de dicho sistema, hicieron que el suelo recuperado se erosione nuevamente, dejando aflorar cangahuas duras y estériles donde solo crece chaparro.

Se demostró que existen dos grupos de propietarios que habilitaron cangahuas para cultivarlas. Los campesinos del primer grupo son aquellos que han elegido, por su propia iniciativa, recuperar su propiedad. Lo hicieron a mano (con pico y pala) o mediante la contratación de los servicios de una excavadora y en muchos casos lo hicieron de ambas formas, cuando los recursos económicos no eran suficientes para permitir hacer toda la labor mecánicamente.

El segundo grupo está constituido por personas que se beneficiaron de programas de asistencia realizados por instituciones públicas (Municipio o Consejo Provincial) o instituciones privadas como ONGs o Fundaciones (IEDECA, SEDAL, Casa Campesina, PACHAMAMA). La fundación PACHAMAMA fue la primera en habilitar cangahuas en esta parroquia hace 25 años, pero sus actividades cesaron hace varios años. Actualmente, el Municipio local puso en marcha proyectos para ayudar a los productores a habilitar mecánicamente sus lotes con cangahua. La asistencia prestada por estas organizaciones es financiera (ayuda para obtener créditos, uso subvencionado de un bulldozer, etc.) y/o técnica para la siembra y manejo de los cultivos en los lotes recuperados. Una de estas recomendaciones es sembrar vicia (Viscia sativa) en combinación con avena (Avena sativa) para incorporación al suelo como abono verde, inmediatamente después de la recuperación.

Se conoce que la habilitación más antigua de cangahua se la lleva a cabo con pico y pala hace más de 40 años, luego la rehabilitación se realizó mayormente en forma mecánica. En la Figura 5 se presenta la información sobre la edad de rehabilitación de las cangahuas y las organizaciones envueltas en el proceso. Además, el uso de maquinaria pesada y/o de un tractor corresponde a habilitaciones hechas desde hace 15 años y se observa una correlación entre la edad de habilitación agrícola, la presencia de las organizaciones no gubernamentales y el tipo de habilitación (Figura 6).

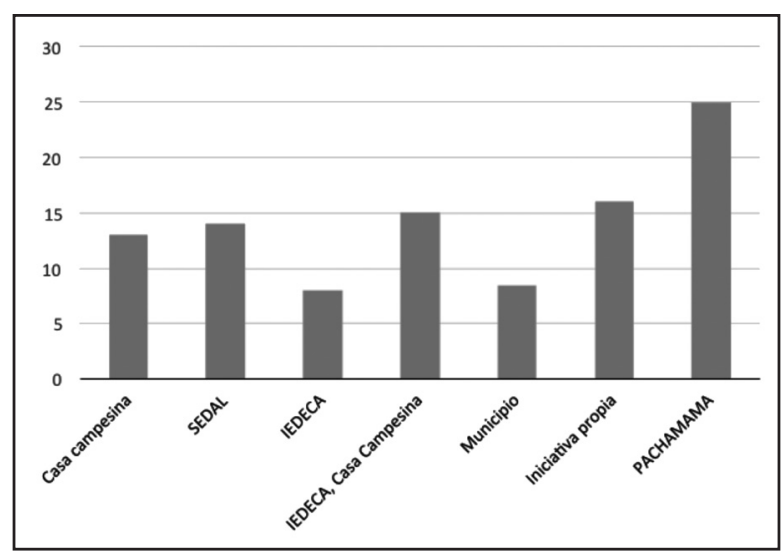

Figura 5. Relación entre la edad promedio de habilitación de las parcelas y las organizaciones envueltas en el proceso. 
Por otro lado, se encontró también que existe una correlación entre la edad de habilitación de las parcelas y el tipo de cultivo sembrado inicialmente. En el caso de las parcelas recuperadas anteriormente, el primer cultivo fue siempre el maíz a pesar de que los rendimientos fueron extremadamente bajos en los 2-3 primeros años de habilitación. El primer cultivo de las parcelas trabajadas durante los últimos años ya no se limita al maíz, que se sigue sembrando en asocio con fréjol, sino que además se siembran abonos verdes (vicia y avena) u otros cultivos siguiendo la recomendación de los organismos envueltos en la rehabilitación.

El procesamiento de las diferentes variables recolectadas en las encuestas mediante correlaciones múltiples encontró para las variables de mayor interés (rendimiento, presencia o no del riego, edad de habilitación de las cangahuas, tipo y sistema de cultivo sembrado) presentaban las siguientes tendencias (Figura 7):

- Las variables que califican rendimiento, tipo de cultivo y tipo de riego tienen alta correlación entre ellas. Esto significa que el riego juega un papel muy importante en el tipo de sistemas de cultivo de la zona del estudio. Los productores que tienen un sistema de riego en sus parcelas tienen cultivos más diversificados y presentan una situación socio-económica más estable que la de los demás campesinos. Por esta razón, la existencia de un sistema de riego es sumamente importante para los productores, e incluso parece esencial para que existan sistemas agroecológicos diversificados en esta área.

- Existe una fuerte correlación entre el sistema de cultivo, edad de habilitación de los lotes y la intervención de las organizaciones que promueven estos trabajos. Esta observación también es de interés ya que confirma las relaciones anteriores. El trabajo de las organizaciones que promueven el mejor manejo de las cangahuas rehabilitadas facilita las operaciones y hace eficiente el uso del suelo mediante la implementación de prácticas agroecológicas por parte de los dueños de las parcelas.

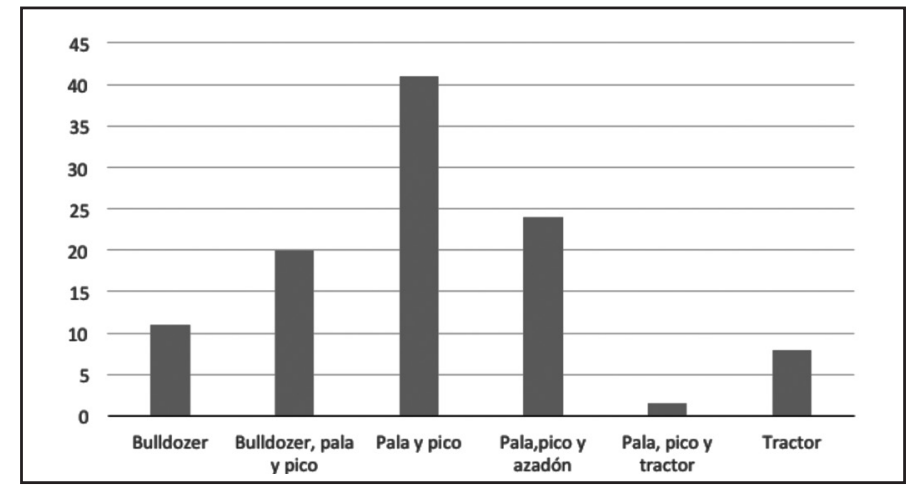

Figura 6. Relación entre la edad promedio de habilitación de la parcela y el modo de habilitación.

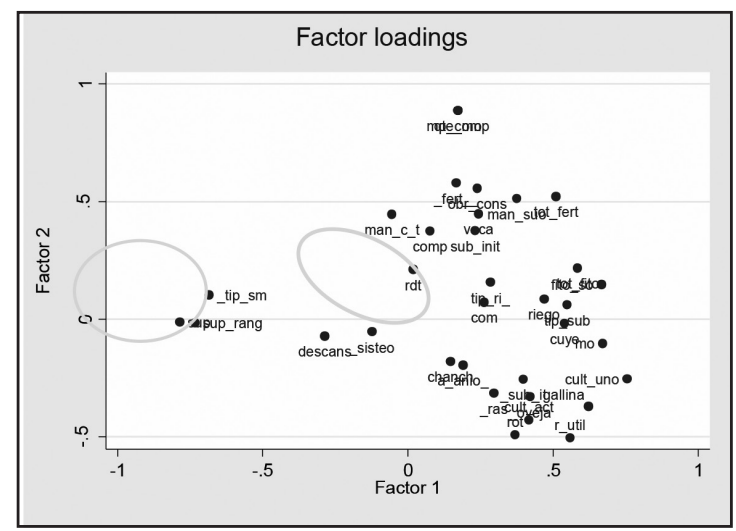

Figura 7. Correlaciones múltiples de las variables recolectadas en el estudio. 


\section{Conclusiones}

La habilitación agrícola de las cangahuas no es solo remover el material. Esta investigación permitió desarrollar el primer diagnóstico del proceso de habilitación de lotes de cangahua por sus dueños. Este proceso de conversión de rocas a suelos que es impulsado por instituciones públicas como los gobiernos locales, por instituciones privadas como las ONGs o fundaciones, e incluso por los propios agricultores, contribuye al desarrollo agrícola y a la estabilización y mejoramiento del paisaje andino. Aunque las superficies habilitadas siguen siendo muy bajas ( 0,6 ha por familia en promedio), se observó que dentro de estas existe un sistema de producción agroecológica que permite un mejor uso de la tierra y que además promueve condiciones sociales, económicas y ambientales sostenibles. Estas parcelas agroecológicas, basadas en la diversificación de producción, en la complementariedad con la ganadería y en diferentes técnicas de cultivo y conservación de suelos, parecen tener todas las condiciones necesarias para garantizar la seguridad alimentaria de los productores de la zona, protegiendo y mejorando rápidamente los suelos. A pesar de que este tipo de experiencia es todavía marginal en el municipio de Cangahua, tanto por el número de familias implicadas como de superficie trabajada, podría servir como modelo de desarrollo agrícola que encaja bien en la propuesta de cambio de matriz productiva, desarrollo sustentable y protección de la salud humana y del ambiente.

Sin embargo, esta propuesta agroecológica es factible únicamente si existe riego y si se implementan prácticas de control de la erosión inmediatamente después de la habilitación de las cangahuas. Las instituciones priorizan el trabajo mecánico de habilitación de las cangahuas, pero frecuentemente se olvidan de promover prácticas de control de la erosión luego de la remoción y ablandamiento del material, si esto sucede el suelo rehabilitado se pierde inexorablemente.

Las recomendaciones actuales se enfocan a promover el incremento rápido de la fertilidad del nuevo suelo, en particular a través de abonos verdes en la primera siembra, así como del uso de abonos orgánicos (estiércol, composta, etc.), prácticas que son aceptadas por los productores porque tienen buenos resultados agronómicos.
Existen otras alternativas de cultivos en el caso de no existir riego. Por ejemplo, en Cayambe y en particular en la parroquia Cangahua, se está implementando la siembra del penco (Agave americana) para extraer y fermentar la sabia y producir chaguarmishqui. Este producto envasado técnicamente se ha incorporado a una cadena local de producción y venta que al momento se está ampliando. La alcaldía de Cayambe, los productores y una pequeña empresa (INDESLAE) están trabajando juntos para rescatar este cultivo y otros productos ancestrales para beneficio de todos los productores.

La sistematización de las experiencias agrícolas de la parroquia Cangahua demostró que existe cierta homogeneidad de las prácticas agrícolas y que en el caso de las zonas con riego, predomina el sistema agroecológico. Si bien estas prácticas son agroecológicas, no lo son por estricta convicción del productor sino porque las circunstancias los obligaron a utilizarlas. En otras palabras, la falta de recursos económicos hace que los campesinos no puedan comprar agroquímicos y por lo tanto no los puedan usar. Sin embargo, esta falta de recursos desarrolla la inventiva y obliga a reciclar. Por esta razón, sería conveniente aprovechar de los trabajos de habilitación de cangahuas para capacitar a los campesinos en las técnicas de producción agroecológica y promover la producción y mercadeo de insumos orgánicos como abonos, compostas y productos biológicos de control de plagas y enfermedades.

Este estudio confirma que es posible y rentable convertir las cangahuas estériles y duras en un suelo fértil y productivo. Para esto es necesario inversión institucional basada no solamente en los trabajos mecánicos de remoción de material endurecido, sino también en una capacitación técnica de los campesinos orientada hacia el desarrollo sistemas agroecológicos sostenibless en las áreas con riego. En las áreas sin riego y con pendientes muy inclinadas es posible desarrollar la agaveforesteria como una alternativa de uso del suelo y de generación de recursos. Para todo esto es fundamental tener un plan de acción, basado en mapa de suelos y dispersión de las cangahuas, localización de las áreas con riego, distribución de la población y de datos agro-socioeconómicos de las zonas que potencialment se podrían intervenir. 


\section{Referencias}

Báez, A. (2008). Formación de agregados y captura de carbono en materiales de origen volcánico de México. México: Campus Montecillo. Postgrado de edafología, p 255. Tesis (Dr. en ciencias).

Benítez D., Navarro H. (1996). Rendimiento de maíz-frijol en un tepetate en el segundo año de rehabilitación agrícola. Los suelos con cangahua en Ecuador. Suelos volcánicos endurecidos. III Simposio Internacional. p 279-286.

Da Costa, M. (2014). Etude exploratoire des modes de mise en valeur agroécologiques en terrains de cangahua habilités dans le nord de la sierra équatorienne, canton de Cayambe. Memoire de fin de curs. Istom-CTI-UCE. París. 83 p.

De Noni G., Viennot M., Asseline J., Trujillo G. (2001). La lutte contre l'érosion dans les Andes Equatoriennes. Terres d'altitude, terres de risque.IRD, 216p. collection latitude 23 .

Doré T., Le Bail M., Martin P. 2006. Système de cultura et territoire. L'agronomie aujourd'hui. France: Quae. 24-27p.

Espinosa, J. (2014). La erosión en Ecuador un problema sin resolver. Siembra un mejor futuro sostenible, 83 años por la soberanía alimentaria de los ecuatorianos. Volumen 1: 56-58.

Flores D., Navarro H. (1996). Manejo agronómico diferencial de la asociación maíz-haba en tepetate de quinto año de uso agrícola. Los suelos con Cangahua en Ecuador. Suelos volcanicos endurecidos, III Simposio Internacional. p287-295.

Flores R. (2014). La agricultura y el desarrollo en Ecuador. Universidad Católica.

Gem B., Cisneros S., Jorge A., Flor I., Trujillo G. (1995). Diagnóstico del contenido de elementos en dos cangahuales de la provincia de Pichincha. Los suelos con Cangahua en Ecuador. Suelos volcánicos endurecidos. III Simposio Internacional. p185-193.

IRR (eds), (2006). Manual de prácticas agroecológicas de los Andes ecuatorianos. Quito, Ecuador.

MAGAP. 2012. Mapas de fertilidad de Suelos.1:250000.

MAGAP geoportail. (2013). [On line]. [06/06/2014] <URL: http://geoportal.magap.gob.ec/inventario. html>

Ministerio del Ambiente. (2013). Beneficios del manejo sostenible de la Tierra, segundo documento técnico.

Parroquia de Cangahua. (2012). Plan de desarrollo y ordenamiento territorial de la parroquia de Cangahua. <URLhttp://www.pichincha.gob.ec/phocadownload/leytransparencia/literal_k/ppot/cayambe/ppdot_cangahua.pdf $>$.

Prat, C. (1996). Erosión, conservación e hidrodinámica. Los suelos con Cangahua en Ecuador. Suelos volcánicos endurecidos. III Simposio Internacional. $p$ 325-330.

Prat, C., Boun-Tieng Lu., Lepigeon I., Faugère G., JeanLouis A. (1996). Los sistemas agropecuarios de producción en tepetates, en cuatro comunidades del altiplano mexicano.

Quantin, P. (2000). L'induraction des matériaux volcaniques pyroplastiques en Amérique Latine: Processus géologiques et pédologiques.Bondy: France ORSTOM.

Salgado R. (2012). Cangahua estudio del entorno natural y social. Quito: Graficas UPEGUI.

Zebrowski C., Azucena V. 1996. El cultivo de la cangahua en el medio campesino tradicional en el Ecuador. Los suelos con Cangahua en Ecuador. Suelos volcánicos endurecidos. III Simposio Internacional. 